\title{
Identification of the death zone: a spatially restricted region for programmed cell death that sculpts the fly eye
}

\author{
JP Monserrate ${ }^{1}$ and C Baker Brachmann ${ }^{\star, 1}$
}

Programmed cell death (PCD) sculpts many developing tissues. The final patterning step of the Drosophila retina is the elimination, through PCD, of a subset of interommatidial lattice cells during pupation. It is not understood how this process is spatially regulated to ensure that cells die in the proper positions. To address this, we observed PCD of lattice cells in the pupal retina in real time. This live-visualization method demonstrates that lattice cell apoptosis is a highly specific process. In all, $85 \%$ of lattice cells die in exclusive 'death zone' positions between adjacent ommatidia. In contrast, cells that make specific contacts with primary pigment cells are protected from death. Two signaling pathways, Drosophila epidermal growth factor receptor (dEgfr) and Notch, that are thought to be central to the regulation of lattice cell survival and death, are not sufficient to establish the death zone. Thus, application of live visualization to the fly eye gives new insight into a dynamic developmental process. Cell Death and Differentiation (2007) 14, 209-217. doi:10.1038/sj.cdd.4401947; published online 19 May 2006

The development of many tissues in metazoan organisms involves an overproliferation of cells balanced with programmed cell death (PCD) to attain the final size. PCD is also an important mechanism for patterning a number of complex tissues by removing cells in specific regions. ${ }^{1-6}$

The Drosophila eye is sculpted by PCD and represents an ideal system to dissect the mechanism of developmentally regulated $\mathrm{PCD} .^{7,8}$ Studies on the fruit fly circumvent some of the difficulties of studying developing organs in mammals, and benefit from the powerful genetics of this model organism coupled with the fact that the cell death pathway is evolutionarily conserved. The $\sim 750$ ommatidia of the fly eye are arranged in a stereotypical hexagonal pattern that is determined in late larval life and refined during mid-pupal development through selective PCD. Prior to the onset of PCD, an excess number of cells lie between ommatidial units; a subset of these seemingly identical cells is removed through cell death, leaving just the right number of cells to solidify the perfect hexagonal lattice of the eye..$^{9,10}$

It is not known how PCD is regulated to ensure that the proper number of cells die. ${ }^{7}$ In contrast to PCD in Caenorhabditis elegans, lattice cell death is not specified by lineage; therefore, cell death in the developing Drosophila retina must be directed by positional information through cell-cell communication, ${ }^{11,12}$ as is also the case in mammalian development. Several laboratories have demonstrated that signaling from the Drosophila epidermal growth factor receptor (dEgfr) $D E R / T o r p e d o / F l b) /$ ras pathway promotes lattice cell life, ${ }^{13-16}$ in part through negative regulation of the cell death effector head involution defective (HID). ${ }^{17,18}$ During the period of lattice cell death, dEgfr is expressed by all lattice cells, and its soluble ligand, Spitz (Spi), is expressed by cone cells and primary pigment cells. ${ }^{13}$ Counteracting the $d E g f r /$ ras pathway is the Notch signaling pathway that directs lattice cell death. ${ }^{13,19}$ Notch is highly expressed in lattice cells. An epistasis experiment demonstrated that Notch acts to promote apoptosis by inhibiting life signaling through $\mathrm{dEgfr}{ }^{20}$

In sum, the current belief is that all lattice cells receive life signals through the $d E g f r / r a s$ pathway with counteracting death signals through Notch. ${ }^{7,20}$ How can these overlapping signals be resolved so that some of the cells die and some live? There are two likely scenarios: (1) life or death signals are propagated directionally, killing cells in specific regions until no cells remain to populate that region and (2) cells die randomly until the right number remain and the death signal is turned off. The distinguishing feature between these two models is the position of the dying lattice cell; scenario (1) is likely to have PCD occurring in stereotypical death regions, whereas in scenario (2) dying cells should be randomly placed. Previous studies using electron microscopy were unable to determine whether certain cell contacts promote life or death among lattice cells. ${ }^{9,10}$

To definitively address where a cell resides when it is instructed to die, we developed a new method to watch the apoptotic process occur in the living pupal eye. Our livevisualization experiments demonstrate that doomed lattice cells are not chosen randomly with respect to their spatial position. Rather, lattice cell PCD occurs in distinct 'death

\footnotetext{
${ }^{1}$ Developmental and Cell Biology, University of California Irvine, Irvine, CA, USA

${ }^{*}$ Corresponding author: CB Brachmann, Developmental and Cell Biology, 5205 McGaugh Hall, Zot 2300, University of California, Irvine, Irvine, CA 9269700 , USA. Tel: 949824 9139; Fax: 949824 1714; E-mail: cbrachma@uci.edu

Keywords: programmed cell death; Drosophila; apoptosis; spatial regulation; Notch; Egf receptor

Abbreviations: PCD, programmed cell death; dEgfr, Drosophila epidermal growth factor receptor; Spi, Spitz; HID, head involution defective; APF, after pupariation formation; CC3, cleaved caspase 3; TUNEL, terminal deoxynucleotidyl transferase-mediated biotinylated UTP nick-end labeling; GFP, green fluorescent protein; E(spl), enhancer of split; P-MAPK, phosphorylated mitogen-activated protein kinase; DN, dominant negative; hs-Gal4, heat shock Gal4; UAS, Gal4 upstream activating sequence

Received 06.12.05; revised 14.2.06; accepted 28.2.06; Edited by S Kumar; published online 19.5.06
} 
zones'. Mechanosensory bristle groups influence the position of the death zone, but are not required for the establishment of a death zone. This novel method sets the stage for detailed studies of the dynamic signaling interplay between cells that results in spatially restricted $P C D$.

\section{Results}

Molecular apoptotic markers cannot distinguish the position of dying cells in the Drosophila retina. Specification of the 14 cells of each ommatidial unit (eight photoreceptor neurons, two primary pigment cells and four cone cells), as well as the mechanosensory bristle groups, begins during larval life and continues through early pupal development. By $18 \mathrm{~h}$ after pupariation formation (APF), the unique morphology of the primary pigment cells, cone cells and bristle groups is evident (photoreceptors lie below the plane of the image, Figure 1a). The remaining cells will form the interommatidial lattice of secondary pigment cells (touching two primary pigment cells) and tertiary pigment cells (touching three primary pigment cells), collectively termed 'lattice cells', that separate neighboring ommatidia and set the precise hexagonal pattern of the adult eye. Over the next few hours of pupal development, the lattice cells and bristle groups reorganize in an ordered fashion, bringing the ommatidia into a tighter configuration in which each is separated by a single layer of lattice cells in an end-to-end chain (Figure $1 b$ and $c$ ). During this time, a few cells per ommatidium are removed through $\mathrm{PCD} .{ }^{21} \mathrm{By} 24 \mathrm{~h}$ APF, the bristle groups have moved to occupy alternating vertices of the lattice (Figure 1c). Excess lattice cells are removed through PCD leaving just one lattice cell to form a secondary pigment cell stretched between tertiary pigment cells and bristle groups (Figure 1d). ${ }^{7,9,10}$

Our studies into spatial regulation of lattice cell death began by investigating whether molecular apoptotic markers identify dying cells early enough in the process to allow us to view their positions. Although both terminal deoxynucleotidyl transferase-mediated biotinylated UTP nick-end labeling (TUNEL) and an antibody that recognizes a Drosophila activated caspase (anti-cleaved caspase 3 (CC3) $)^{22}$ label dying lattice cells, neither could resolve the 'starting' position of the apoptotic cells because by the time these methods identified dying cells, they had lost their apical contacts and shrunk basally (Figure 1e and f; see also Supplementary Figure 1).

Live visualization of the developing pupal retina unambiguously identifies dying cells. Ultrastructural analyses of the pupal eye demonstrated previously that a dying lattice cell loses its apical foothold before undergoing other structural changes. ${ }^{9,10}$ Therefore, we needed to develop a method to identify dying cells before or at this point to definitively position the doomed cell before it dies. We took advantage of the fact that once the eye disc has everted in early pupal life, it is accessible to microscopic evaluation. Expression of a membrane-localized green fluorescent protein (GFP) molecule allowed visualization of the cells in the living pupal eye. Through time-lapse imaging, capturing a Z-series at each time point, the live-visualization
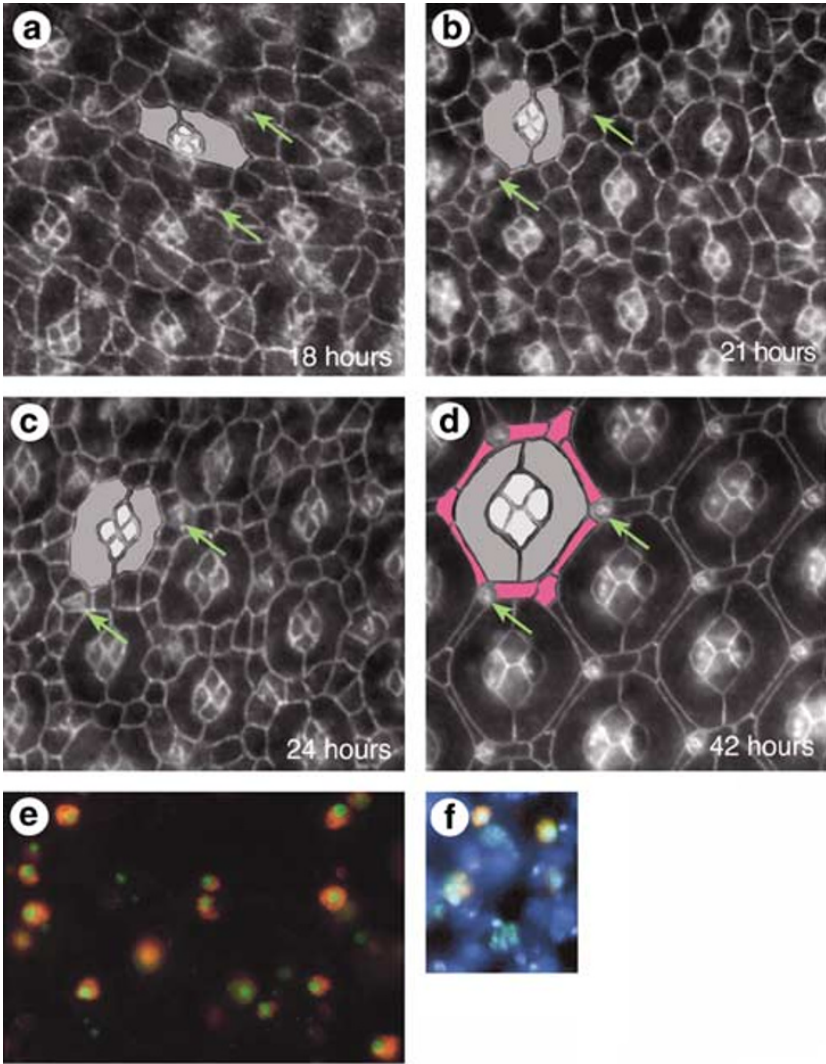

Figure 1 Apoptosis shapes the pupal eye. (a-d) Whole-mount pupal retinae dissected at developmental times are shown, immunostained with anti-armadillo. For clarity, an ommatidium is shaded in each panel. The primary pigment cells are dark gray, cone cells are light gray and examples of bristle groups are indicated by green arrows. Interommatidial lattice cells account for the remainder of cells. (a) $18 \mathrm{~h} \mathrm{APF}$, (b) $21 \mathrm{~h} \mathrm{APF}$, (c) $24 \mathrm{~h} \mathrm{APF}$, (d) $42 \mathrm{~h} \mathrm{APF.} 1^{\circ}$, primary pigment cells; cc, cone cells; $2^{\circ}$, secondary pigment cells; $3^{\circ}$, tertiary pigment cells. Collectively, the $2^{\circ}$ and $3^{\circ}$ cells are referred to as the interommatidial lattice (pink). (e, f). Lattice cell death is apoptotic, but dying cells cannot be positioned using molecular markers. (e) TUNEL labeling of apoptotic nuclei in green and CC3 immunostaining in red of a $26 \mathrm{~h}$ APF pupal retina demonstrating that dying cells are small bodies that are TUNEL positive and CC3 reactive. (f) Higher magnification view of another disc demonstrating that shrunken, dying cells lie in the lattice cell nuclear layer and that fragmented nuclei are still TUNEL positive but may no longer be CC3 reactive (DAPI, blue; TUNEL, green; CC3, red). Anterior is to the left

method unmistakably discerned dying cells as they lose their apical footprint and begin to shrink below the surface. An example of a series of time-lapse images is shown in Figure 2a (discussed below). Thus, the live-visualization method fulfills the requirement of identifying dying cells at the first structural indication of death.

Cells die in very specific positions within the interommatidial lattice - the 'death zone'. We carefully analyzed a visual field from three pupal retinae and tabulated the location of each apoptotic cell with respect to the neighboring ommatidia (roughly 130 apoptotic events per retina). The results are presented in a graph that depicts the mean of the three experiments (Figure 2b). The time course shown in Figure $2 \mathrm{a}$ is a randomly chosen region from one of these retinae (the schematic defines horizontal and oblique regions as they are used throughout this study). Remarkably, 


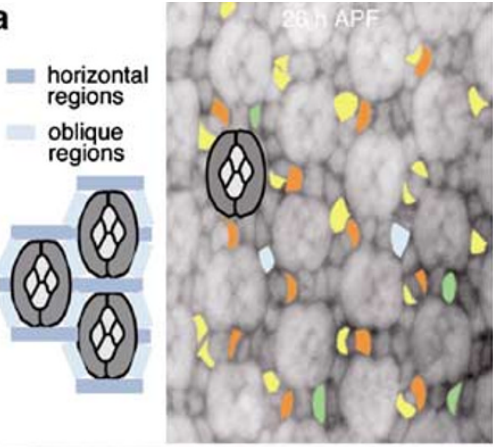

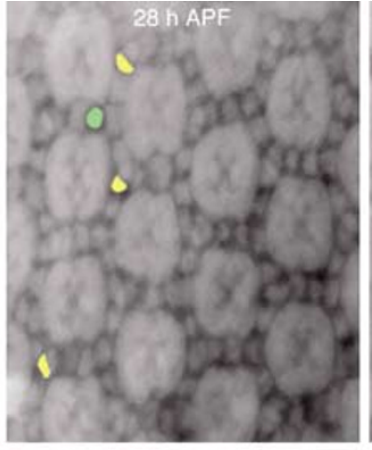
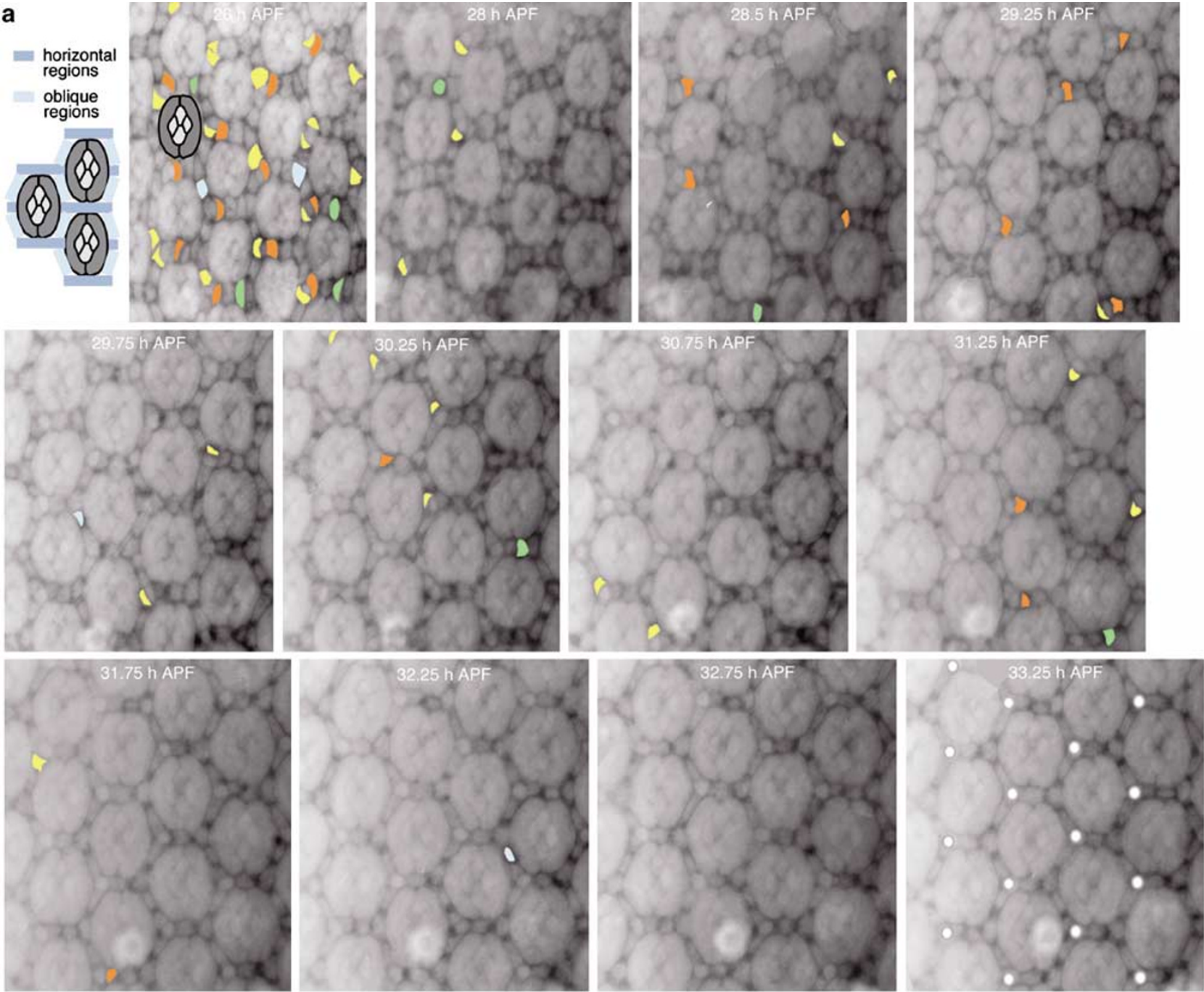

b

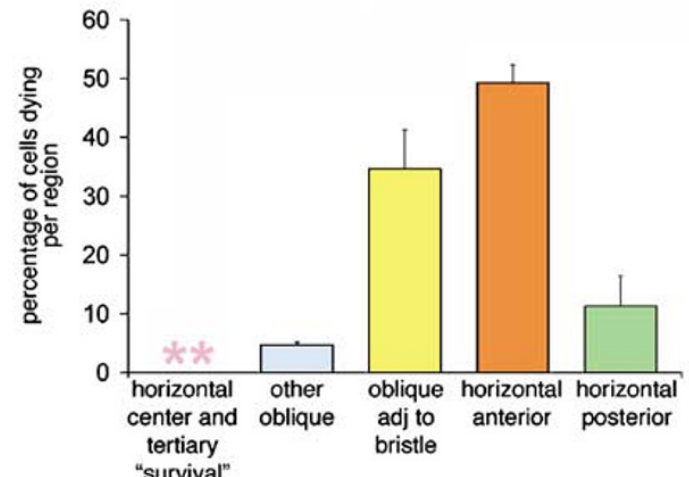

c

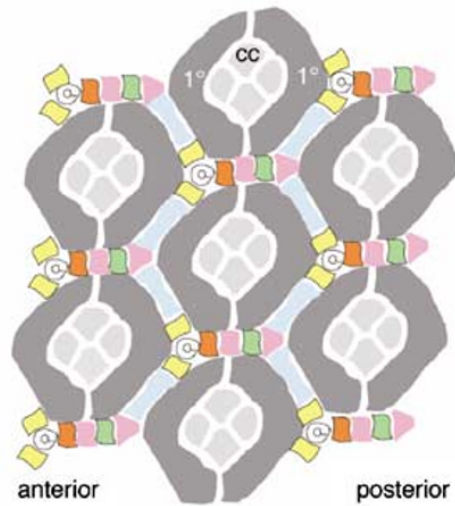

Regions:

horizontal anterior

口

$\square$ oblique adjacent to a bristle group

other oblique

survival positions:

horizontal center and tertiary

(a) bristle group

Figure 2 Lattice cell apoptosis is spatially regulated. Time-lapse images of the developing pupal retina, beginning at $26 \mathrm{~h} \mathrm{APF}$. (a) The image is inverted so that the GFPlabeled membranes are black. The increase in size of the imaged region is owing to pupal development. Dying cells are highlighted on the image just prior to their demise. Cells that die in the horizontal anterior region are in orange. Cells that die in oblique regions adjacent to bristle groups are yellow and the few that die in the horizontal posterior and other oblique regions are green and light blue, respectively. On the first image, all cells that will die over the time course have been identified in the appropriate color. The bristle groups are apparent from the earliest time point at positions close to their final positions and are identified by white circles on the final image. An ommatidial unit has been drawn in for orientation. (b) Percentage of cells observed dying graphed relative to specific regions. Colors correlate to those used in (a) and (c). Pink asterisks highlight that no cells died in these regions. (c) Schematic of the pupal retina, colors correlate with (a) and (b). Each colored rectangle corresponds to a region in which cells will either be more likely to live (pink) or die (orange and yellow). Note that cells that die adjacent to a bristle group may die above or below the bristle group as drawn. Live imaging of the pupal eye had no effect on the viability of the pupa nor did it lead to any visible morphological change in the adult eye. Anterior is to the left 
roughly one-half of all apoptotic cells occupy a horizontal anterior position as they begin to undergo death (orange in Figure $2 a-c)$. Another large percentage of dying cells (35\%) occupies an oblique position adjacent to a bristle group (yellow in Figure $2 \mathrm{a}-\mathrm{c}$ ). In contrast, dying cells in the other oblique regions (light blue) or horizontal posterior position (green) account for only $5 \%$ and $11 \%$ of apoptotic cells, respectively (Figure $2 \mathrm{a}-\mathrm{c}$ ). Previous electron microscopy studies had noted that many dying cells were located near bristle groups; ${ }^{9,10}$ our results confirm this and solidify the observation by quantifying it. The two regions, horizontal anterior and oblique adjacent to a bristle group, are therefore defined as the 'death zone' where $85 \%$ of apoptotic lattice cells will be located.

The time-lapse images reveal that dying cells are replaced by adjacent lattice cells that will, in turn, die should too many cells remain in the region. Additionally, these experiments identify survival positions. No cells die in the tertiary position or in the horizontal face if contacting all four primary pigment cells (survival positions are pink in Figure 2c, see also Supplementary Figure 2). By the end of the period of PCD, these horizontal center cells will stretch to occupy the horizontal face between the tertiary cell and the bristle group. Thus, the live-visualization method is the first to unambiguously demonstrate that PCD in the pupal eye is nonrandom.

Bristle groups influence the placement of the death zone. Because apoptotic cells were often found next to the bristle group, we next investigated whether lack of bristle groups would alter the pattern of lattice cell apoptosis. Flies ectopically expressing wingless in the eye ( $\mathrm{P}[\mathrm{sev}-w g]$ flies) have normal eyes except that mechanosensory bristle groups are replaced with tertiary cells ${ }^{23}$ (Figure 3a). We confirmed the absence of bristle groups (Figure $3 \mathrm{~b}$ and $\mathrm{c}$ - compare with Figure $3 \mathrm{~d}$ ). Because ectopic $\mathrm{Wg}$ protein in the pupal eye might influence lattice cell apoptosis, we investigated whether the sev promoter drives expression of $w g$ in the mid-pupal $\mathrm{P}[s e v-w g]$ eye. No $\mathrm{Wg}$ protein expression was observed in $\mathrm{P}[\mathrm{sev}-w g]$ or control wild-type retinae at 24 or $28 \mathrm{~h}$ APF (data not shown).

Live imaging was employed to investigate whether removal of bristle groups influenced the placement of the death zone. The lack of bristle groups had a dramatic effect on the pattern of dying cells in the pupal retina (Figure $3 f$ and $g$ ). The vast majority of dying cells (80-90\%) occupy a position either posterior (green) or anterior (orange) in the horizontal face between ommatidia. In contrast to eyes with bristle groups, few cells die in oblique positions (light blue). Thus it is clear that, without bristle groups, the death zone moves to encompass both anterior and posterior regions of the horizontal face, straddling the horizontal center 'survival' position, and is no longer associated with oblique positions. This result establishes that the bristle group, although not required for patterning of the eye through apoptosis, influences the spatial placement of the death zone.

Signaling through the Drosophila Egfr and Notch receptors is active in all lattice cells. Loss of Notch signaling leads to ectopic life of lattice cells. ${ }^{13,19}$ Conversely, a block in dEgfr signaling leads to ectopic death of lattice
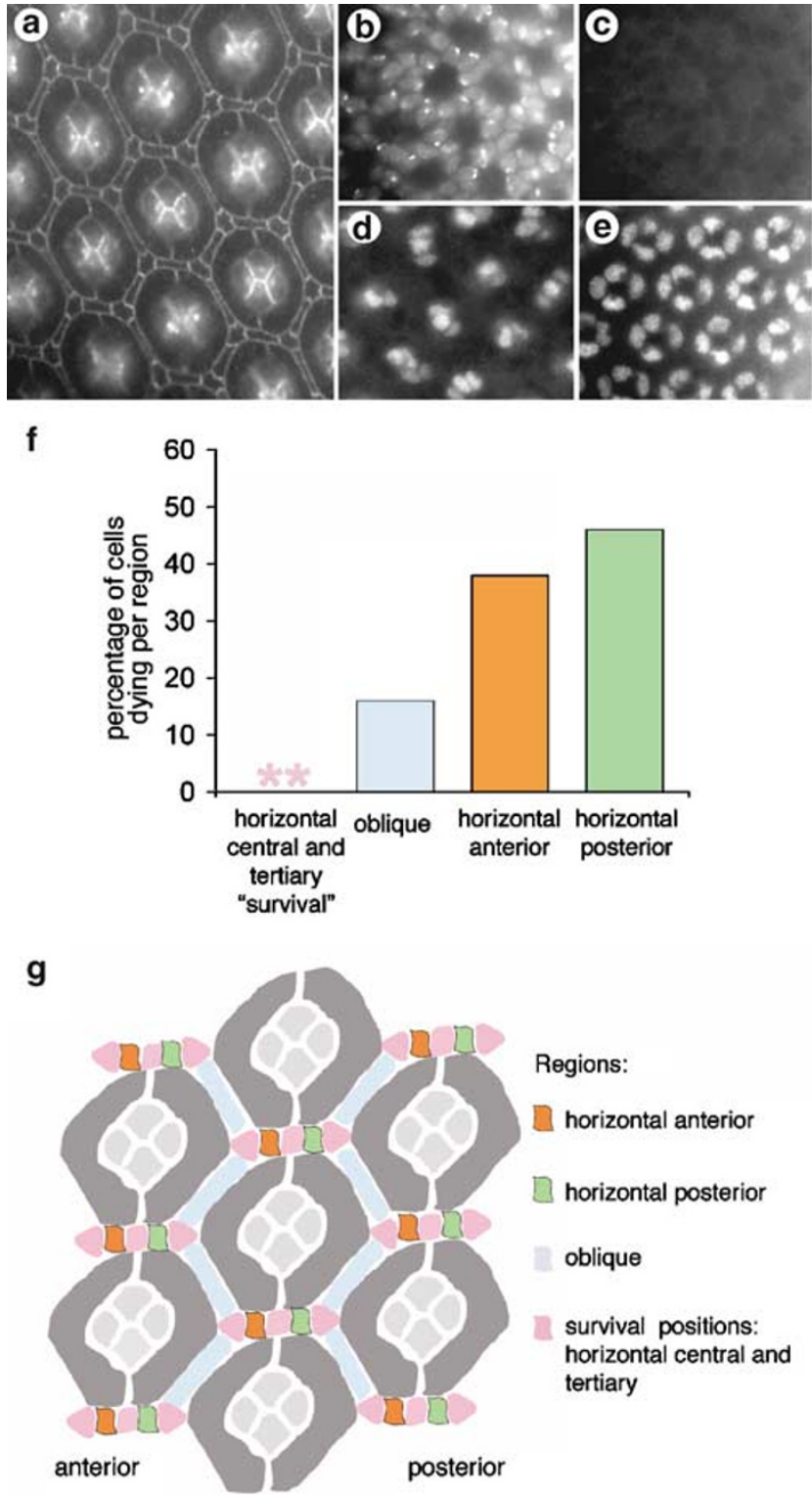

Figure 3 Spatial regulation of lattice cell PCD is altered in the absence of bristle groups. Characterization of the $P$ [sev-wg] retina. (a) Anti-armadillo immunostain of a $42 \mathrm{~h}$ APF P $[$ sev-wg] retina showing no patterning defects and a replacement of all bristle groups with tertiary cells. (b, c) DAPI staining of $26 \mathrm{~h}$ APF P[sev-wg] eye disc identifying lattice cell nuclei (b) and lack of immunostaining with an antibody specific for cut (c). All four bristle nuclei of a wild-type $26 \mathrm{~h}$ APF pupal eye disc are identified by anti-cut (d). (e) Cone cell nuclei are cut-positive in $\mathrm{P}[\mathrm{sev}$-wg] retina from (b) and (c), as an internal control for the antibody used. (f) Percentage of cells observed dying graphed relative to specific regions in $\mathrm{P}[$ sev-wg] retinae demonstrating that $\sim 85 \%$ of cell death occurs in two specific regions, but in contrast with wild type, these two regions are now both in the horizontal face. Colors correlate with those in Figure 2 and in $(\mathbf{g})$. Pink asterisks highlight that no cells died in these regions. Shown is the average of two experiments. (g) Schematic of the pupal $P[s e v-w g]$ retina. Colors correlate with (f). Anterior is to the left

cells, whereas hyperactivation of dEgfr signaling rescues all lattice cells from death. ${ }^{13-16}$ As we determined that apoptosis of lattice cells is spatially restricted to the death zone, we hypothesized that we could visualize changes in Notch or dEgfr signaling in lattice cells that are destined to die. Specifically, we expected to see a reduction in dEgfr 
signaling or an induction of Notch signaling in cells in the death zone regions: horizontal anterior or oblique adjacent to bristle groups. We chose the Notch target gene, enhancer of split $(\mathrm{E}(\mathrm{spl}))$, as a marker for active Notch signaling ${ }^{24}$ and phosphorylated mitogen-activated protein kinase (P-MAPK) as an indicator of active dEgfr signaling. To show specificity for the anti-E(spl) and anti-P-MAPK antibodies used, we ectopically expressed dominant-negative (DN) dEgfr
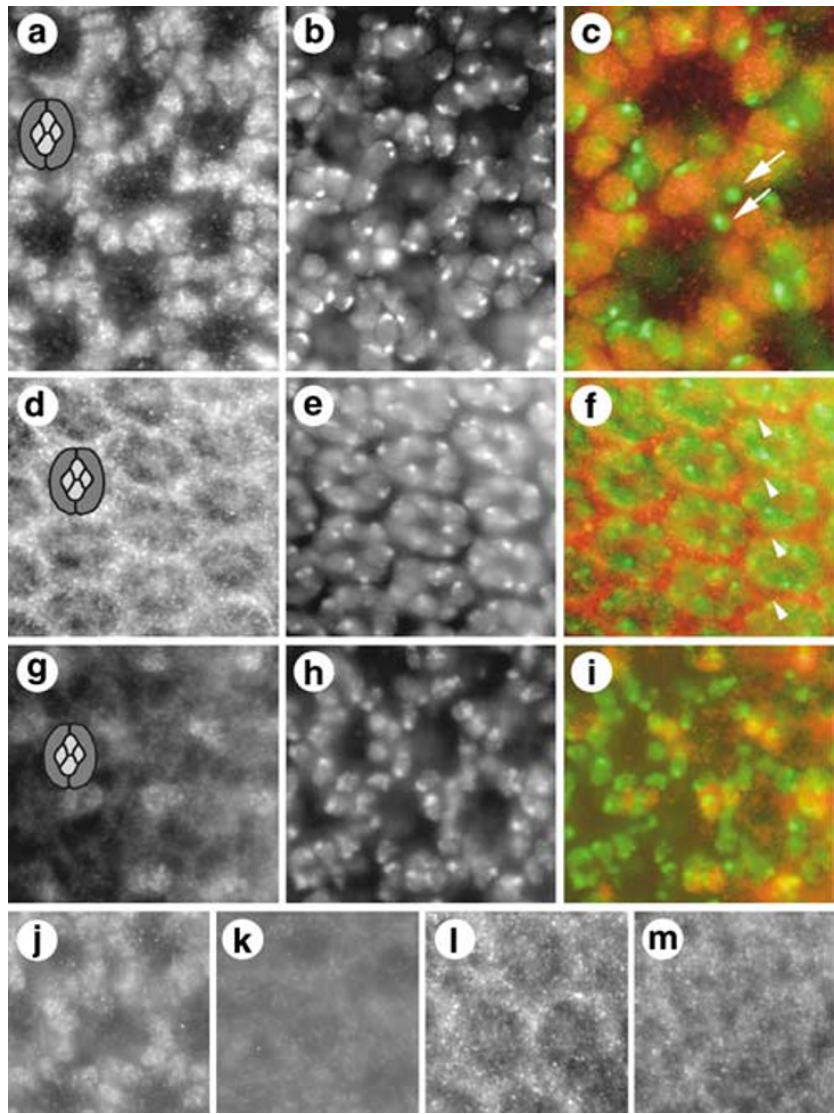

Figure 4 Lattice cells transduce Notch and dEgfr signals. (a-c) Notch signaling as determined by $E(s p l)$ immunostaining. (a) $E(s p l)$ immunostaining of lattice cell nuclei. (b) DAPI of the same region as in (a). (c) Close-up view of a different region demonstrating that dying nuclei are not $\mathrm{E}(\mathrm{spl})$ positive (arrows); nuclei in green, $\mathrm{E}(\mathrm{spl})$ in red. Thus, $\mathrm{E}(\mathrm{spl})$ may be a caspase cleavage substrate. Healthy nuclei that are $E(s p l)$ negative are bristle group nuclei. (d-i) $d E g f r$ signaling as determined by an antibody specific for P-MAPK (anti-dpERK). (d) Lattice cells contain cytoplasmic P-MAPK. (e) DAPI of the same region as in (d). (f) Overlay of (d) and (e); nuclei in green, P-MAPK in red. The bristle group does not maintain cytoplasmic P-MAPK (white arrowheads mark a few bristle groups). (g) P-MAPK immunostaining demonstrating that lattice cell nuclei are negative and that at least two of the bristle group nuclei contain P-MAPK (the bristle nuclei are above the plane of the lattice cell nuclei and thus, slightly out of the focal plane). (h) DAPI of the same region as in (g). (i) Overlay of (g) and (h); nuclei in green, P-MAPK in red. Ommatidial schematics are included in (a), (d) and $(\mathbf{g})$ to orient the reader. Anterior is to the left. (j, $\mathbf{k})$ Reduction in $\mathrm{N}$ signaling by ectopic expression of a dominant-negative Notch, $\mathrm{N}$ ECN substantially reduces $E(s p l)$ reactivity. (j) $E\left(\right.$ spl) immunostaining of $h s-N^{E C N}$ retina, not subjected to heat shock. (k) $\mathrm{E}(\mathrm{spl})$ immunostaining of $h s-N^{E C N}$ retina $1 \mathrm{~h}$ after heat shock. $(\mathrm{I}, \mathrm{m})$ Reduction in dEgfr signaling by ectopic expression of a DN dEgfr greatly reduces antibody reactivity for P-MAPK. (I) P-MAPK immunostaining (antidpERK) of $h s-D N-d E g f r$ retina, not subjected to heat shock. (m) P-MAPK immunostaining of $h s-D N-d E g f r$ retina 45 min after heat shock. Note: For each pair ( $\mathbf{j}$ and $\mathbf{k}, \mathbf{I}$ and $\mathbf{m}$ ), the same exposure time was used for imaging (hs $D N-d E g f r)$ or DN Notch (hs-N $N^{E C N}$ ) to reduce signaling of each pathway (Figure 4j-m). At $26 \mathrm{~h}$ APF, each visual field in the following experiments investigates a number of dying cells as well as those preparing to die (see Figure 1e and Supplementary Figure 1).

The antibody specific for $\mathrm{E}(\mathrm{spl})$ established that all lattice cells of $26 \mathrm{~h}$ APF fixed pupal retinae robustly express $E(s p l)$, indicating an activated Notch signaling pathway in all lattice cells (Figure $4 a$ and b). Dying, condensed nuclei lose $E(s p l)$ immunoreactivity, suggesting that $E(s p l)$ may be a caspase target (Figure 4c). Similarly, the antibody specific for P-MAPK (anti-dpERK) demonstrated that all lattice cells maintain activated MAPK at similar levels regardless of position (Figure 4d-f). Intriguingly, only cytoplasmic P-MAPK was detected in the lattice cells, although at least two of the bristle group cells display nuclear P-MAPK and not cytoplasmic P-MAPK (Figure 4f, arrowheads and $g-i)$. This suggests that dEgfr signaling does not modulate transcription of nuclear targets, but instead leads to cytoplasmic P-MAPK.

Because P-MAPK is directly regulated by dEgfr signaling, we expected to see a reduction in P-MAPK levels in cells signaled to die. We did not see this (although a slight change in P-MAPK levels or a qualitative change in the protein would not be detected), implying that loss of life signaling through dEgfr is not the apoptotic inducer. Yu et al. ${ }^{20}$ proposed that survival of lattice cells is promoted by inhibition of Notch activation, preventing Notch antagonism of dEgfr activity. Despite the fact that $\mathrm{E}(\mathrm{spl})$ protein may not respond to rapid changes in Notch signaling, our data clearly refutes this idea because all lattice cells, whether living or dying, are transducing a Notch signal.

Spitz secreted from cone cells and primary pigment cells rescues lattice cells from death. Alongside our experiments investigating dEgfr signaling, we tested the proposed model that sSpi from cone cells and primary pigment cells could block apoptosis in lattice cells. We investigated which cells can influence lattice cell death through Spi secretion using the 'FLP-out' technique 25,26 to ectopically express sSpi or mSpi (the full-length molecule) in random cells of the pupal retina. Expression of $\mathrm{mSpi}$ from single cells in the retina had no effect on retinal patterning (data not shown), consistent with other experiments in which $\mathrm{mSpi}$ expressed in the eye was inactive owing to lack of processing. ${ }^{16,27}$

In instances where a single lattice cell expressed sSpi, lattice cells multiple cell diameters from the sSpi-expressing cell were rescued from death, as expected from a secreted ligand that moves outward symmetrically in all directions (Figure 5a and b). Note, however, that it is unlikely that sSpi is the spatial regulator as (1) sSpi presumably moves radially outward and (2) experimentally determined levels of P-MAPK (Figure 4) indicate that all lattice cells receive the same level of dEgfr ligand.

Spi secreted from primary pigment cells rescues lattice cells nearby (Figure 5d). Additionally, sSpi produced by cone cells can traverse the primary pigment cell, leading to ectopic lattice cells (Figure $5 \mathrm{c}$ ). Because sSpi was turned on in the marked cells as the end-to-end rearrangement was beginning, the 
pattern of rescued lattice cells may reflect a group of cells that were clustered.

Rescued lattice cells appear to be attracted toward the sSpi cell (refer to Figure $5 a$ and b); thus, sSpi may be important for the normal end-to-end reorganization of lattice cells seen at $\sim 24 \mathrm{~h}$ APF. Occasionally, we observed very small cells in the death zone that expressed sSpi (Figure 5e). These cells may have been signaled to die, but were subsequently rescued by sSpi expression (expressed from $21 \mathrm{~h} \mathrm{APF}$ onward). This intriguing observation suggests that the cell shape change occurs before the point-of-no-return in cell death and implies that increased P-MAPK signaling can stop the apoptotic process once it has begun.

Lattice cells that reside in the death zone prior to the period of cell death have a lower dEgfr signaling threshold. We next investigated how blocking of dEgfr
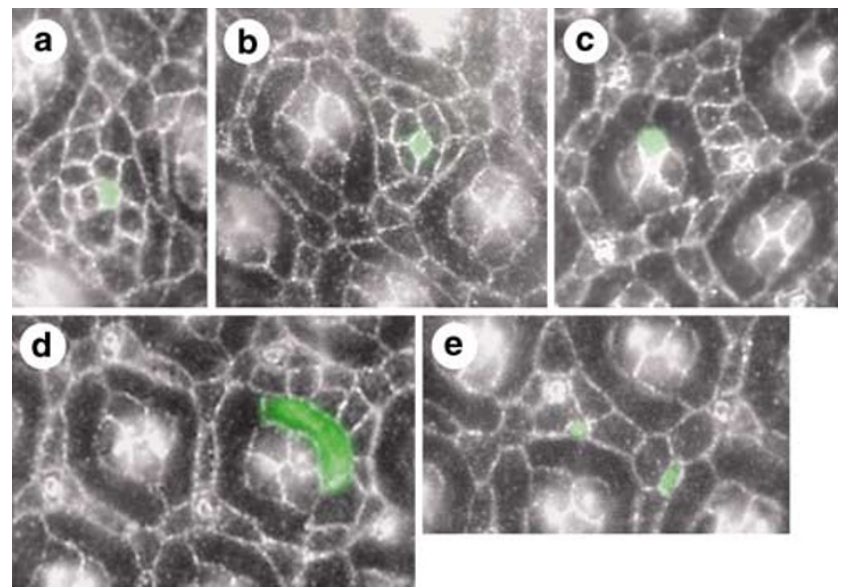

Figure 5 Secreted Spitz rescue cells from PCD several cell diameters away. 'FLP-out' experiments to constitutively drive expression of sSpi from single cells. $h s$ FLP; Act5C $>y^{+}>$Gal4/+; UAS-sSpi/ + pupae were heat shocked at $37^{\circ} \mathrm{C}$ as described for each and dissected at $42 \mathrm{~h} \mathrm{APF}$. Anti-armadillo immunostains with constitutive sSpi-expressing cells identified by coexpression of GFP (green). (a, b) From heat shock at $5.75 \mathrm{~h} \mathrm{APF}$ for $5 \mathrm{~min}$. (c, d, e) From heat shock of $21 \mathrm{~h} \mathrm{APF}$ pupa for $20 \mathrm{~min}$

Figure 6 Lattice cells with reduced dEgfr signaling can survive, whereas lattice cells in the death zone reveal a sensitivity to reduced dEgfr signaling. (a-c) 'FLPout' experiments to constitutively express DN-dEgfr in individual cells. (a) Antiarmadillo outlines membranes of cells in a hs-FLP; Act5C $>y^{+}>$Gal4/UAS-DNdEgfr; UAS-DN-dEgfr/ + retina heat shocked at $20 \mathrm{~h} \mathrm{APF}$ for $5 \mathrm{~min}$ at $37^{\circ} \mathrm{C}$ and dissected at $42 \mathrm{~h}$ APF. Note that occasional lattice cells are missing. (b) Cells constitutively expressing DN-dEgfr are identified by coexpression of GFP. (c) Overlay of (a) and (b) with GFP in green. (d) lattice Gal4; UAS-DN-dEgfr retina demonstrating loss of lattice cells and occasional primary pigment cells (lattice cell Gal4 drives in a subset of photoreceptor cells and peripodial cells in the larval eye, but expresses primarily in lattice cells with occasional weak expression in primary pigment cells in 24-26h APF pupal retinae (data not shown)). (e-g) Temporal expression of DN-dEgfr using $h s-G a l 4$, each heat shocked $40 \mathrm{~min}$ at $37^{\circ} \mathrm{C}$ at $23.5 \mathrm{~h}$ APF. (e) $h s-G a l 4$ control retina. (f, g) Regions from two different $h s-G a l 4, U A S-D N$ $d E g f r$ retinae. Death zone lattice cells are more sensitive to reduction in $\mathrm{dEgfr}$ signaling, regardless of whether bristle groups are present. (h) A hs-Gal4, UAS-DNdEgfr retina heat shocked for $80 \mathrm{~min}$ at $37^{\circ} \mathrm{C}$ at $24 \mathrm{~h} \mathrm{APF}$, note the almost complete loss of lattice cells with the longer heat shock. (d-h) Anti-armadillo immunostains signaling in individual cells would affect lattice cell apoptosis. Using the FLP-out technique, a dominant-negative form of dEgfr (DN-dEgfr, ${ }^{16}$ ) was expressed in random single cells exclusively during the time of death. Unexpectedly, we observed that individual lattice cells expressing high levels of DN-dEgfr are still able to populate the fully formed lattice (Figure $6 a-c)$. This is in opposition to data in which global
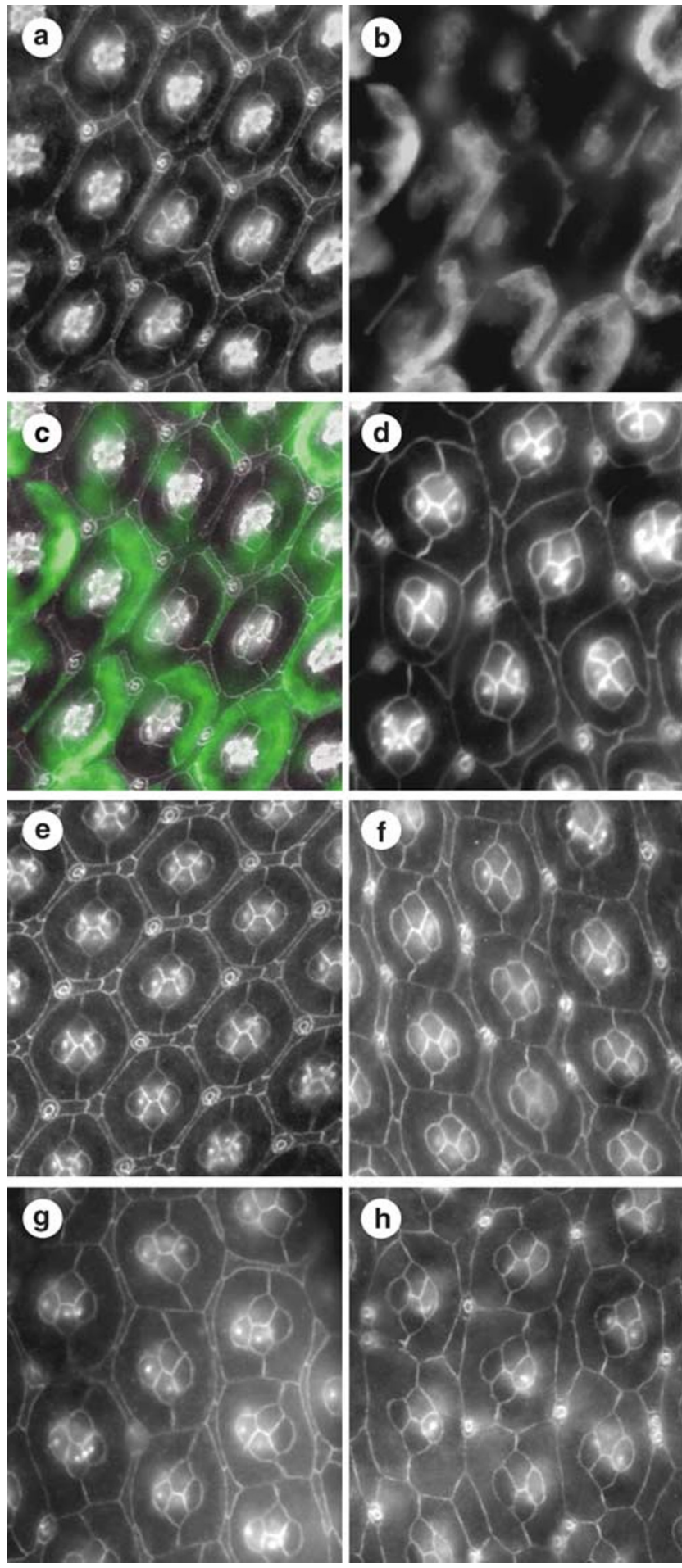
expression of $D N$-dEgfr was shown to lead to cell death in virtually all lattice cells ${ }^{16}$ (and see Figure 6h). In our experiments, we also observed patterning defects indicative of excessive cell death. This suggests that individual lattice cells have differing thresholds for life signaling.

We reasoned that a major difference between our FLP-out experiment and previous experiments that reduced dEgfr signaling in the entire retina was that we did not alter dEgfr signaling in any cells that neighbor a lattice cell expressing DN-dEgfr. Therefore, we ectopically expressed DN-dEgfr primarily in all lattice cells with the result that almost no lattice cells survived (Figure $6 \mathrm{~d}$ ). This demonstrates that when dEgfr signaling is removed from all lattice cells, extensive apoptosis occurs. In contrast, removal of dEgfr signaling in single cells does not necessarily lead to that cell's death.

We hypothesized that a combination of signals regulated temporally might dictate whether a cell is sensitive to loss of dEgfr signaling. Therefore, we activated expression of $D N$ dEgfr in short pulses during pupal development (Figure $6 \mathrm{e}-\mathrm{h}$ ). Strikingly, a pulse of $40 \mathrm{~min}$ at $23.5 \mathrm{~h}$ APF specifically removed lattice cells in the horizontal face, leaving the precise number of oblique lattice cells in proper positions (Figure $6 f$ and g). At this time point, no lattice cells have achieved the survival position of touching four primary pigment cells. A longer pulse resulted in loss of all lattice cells (Figure 6h). Thus, lattice cells that reside in the death zone are more sensitive to reduction in dEgfr signaling.

\section{Discussion}

PCD occurs in the death zone. We have used the Drosophila pupal retina as a model system to study spatial regulation of $P C D$. Apoptosis of a subset of lattice cells in the mid-pupal retina is the final patterning step in sculpting the fly eye. The static nature of ultrastructural studies made it impossible to determine whether dying lattice cells occupied specific positions between ommatidia. ${ }^{9,10}$ Live visualization of the pupal retina is advantageous because it not only allows snapshots to capture specific events but also allows one to identify an apoptotic cell, and essentially look back in time to assign the cell's position before death.

Using time-lapse imaging of the eye of the living pupa, we identified dying cells as they lost their apical footprint, one of the earliest death events. We determined that $50 \%$ of apoptotic lattice cells occupied a position next to the bristle group and in the horizontal face between ommatidial units. Furthermore, $35 \%$ of dying lattice cells were located next to the bristle group along an oblique face. Thus, a remarkable $85 \%$ of apoptotic lattice cells died in two very specific regions between ommatidial units and adjacent to bristle groups.

However, bristle groups cannot be the origin of the death signal as removal of bristle groups does not block apoptosis. Instead, in the absence of bristle groups, the pattern of lattice cell death changed dramatically. Lattice cells at either the anterior or posterior portion of the horizontal face now had roughly an equal chance of dying. Together, the two horizontal regions accounted for $85 \%$ of the lattice cells undergoing PCD. We conclude that lattice cell PCD is regulated spatially so that apoptosis removes cells occupying the anterior and posterior horizontal positions between ommatidia. We have termed this region the 'death zone'. Between these two points of position-specific PCD, a single cell is protected from apoptosis. Apoptosis in the death zone ends when only this cell remains in the horizontal face between ommatidia.

How could bristle groups so dramatically affect the pattern of PCD? One possibility is that the bristle groups attract cells to their death in the death zones. Miller and Cagan ${ }^{13}$ showed that bristle groups secrete Spi and our data (Figure 5) demonstrate that lattice cells respond by surrounding the source of secreted Spi. Just prior to the cell death period, there are on average 1.6 times more cells in the regions around the bristle groups than in other vertices (CBB, unpublished results). Additionally, in the absence of bristle groups, more cells appear to populate the horizontal regions prior to death (at the expense of the oblique regions).

The Notch and Egfr signaling pathways in pupal retinal PCD. Survival of lattice cells depends on a signal, hypothesized to be secretion of Spi, from primary pigment cells and/or cone cells. ${ }^{13}$ Our experiments confirm that lattice cells transduce a dEgfr signal, but the interpretation is complicated by the fact that we were unable to detect P-MAPK in lattice cell nuclei, suggesting that none of the cells respond transcriptionally to dEgfr signaling. Instead, we identified P-MAPK in the cytoplasm of all lattice cells, including those in the death zone. It was recently reported that retaining P-MAPK in the cytoplasm in cells in the larval eye disc is important for normal development. ${ }^{28,29}$ In the current study, it is unlikely that blocking of nuclear MAPK signaling by 'cytoplasmic hold' is required to induce apoptosis because ectopic transcriptional repression of activated MAPK targets by expression of the activated form of aop had no effect on apoptosis in the pupal retina (CBB, unpublished results). Taken together, these experiments argue that dEgfr signaling in lattice cells serves solely to activate P-MAPK in the cytoplasm. Cytoplasmic P-MAPK may suppress apoptosis through phosphorylation of cytoplasmic proteins such as HID.

In light of the fact that Notch is upstream of dEgfr, a new model was put forth: primary pigment cells and/or cone cells promote survival by inhibiting Notch activation and its disruption of dEgfr signaling in lattice cells. ${ }^{20}$ We were able to directly test this model because our live-visualization data predicted the pattern of death signals. Our data do not support this hypothesis as all lattice cells, whether living or dying, are transducing a Notch signal; surviving lattice cells show no downregulation or inhibition of Notch signaling. We instead propose that Notch dampens the Spi-activated dEgfr survival signaling in all lattice cells. Thus, experimental removal of Notch (i.e. heat shock of $N^{t s 1}$ ) would cause a general increase of dEgfr signaling (and survival of all cells). Conversely, loss of dEgfr signaling would lead to PCD, the default pathway. This model predicts that Notch transcriptionally activates a gene whose protein product keeps dEgfr signaling poised. A second scenario is one in which the Notch protein may itself influence the life or death decision of a cell. That Notch acts through a noncanonical pathway has been suggested previously. ${ }^{30-33}$ In this model, Notch might function to activate 
PCD only in death zone cells, acting as a third signal as described below.

Cell-cell signaling defines the death zone. How is the death zone molecularly defined? Cells in the horizontal region are more sensitive to a reduction of dEgfr signaling, but, as demonstrated by P-MAPK levels, this is unlikely to be owing to a lower level of dEgfr signaling. Thus, if the death zone is defined by dEgfr signaling, this may be owing to a qualitative change in cytoplasmic P-MAPK activity. A more likely possibility is that dEgfr signaling (dampened by Notch) merely poises the cell to allow a third signal to induce PCD specifically in the death zone. In support of this idea is the finding that cells in the death zone regions are more likely than cells in other regions to die in response to a brief interruption in dEgfr signaling.

Localization of apoptosis in the lattice to death zone regions brings additional complexity to the problem of PCD regulation. Clearly, the signal that regulates this final cell fate must be localized. The most likely candidate cell to produce the regulating signal is the primary pigment cell. However, each primary pigment cell contacts numerous lattice cells; some that will survive and some that will die. Thus, we suggest a model in which the dorsal and ventral regions of each primary pigment cell localize a death-inducing factor. Evidence for such a subcellular domain has been seen in primary pigment cells (CBB, unpublished results). The contact regions at which the two primary pigment cells meet must either block this signal or localize a strong survival signal.

Placed in the context of other examples in which the architecture of a tissue is shaped through selective PCD, the Drosophila retina is a unique model in that it can be manipulated genetically, microscopically and development can be visualized in the living animal. The determination that apoptosis is spatially regulated in the pupal eye directs future experiments designed to identify how the death zone is created.

\section{Materials and Methods}

Drosophila strains. Stocks used were: UAS-gap43GFP on II (gitt of P. Garrity, MIT), P[sev-wgl/TM3 (gift of K. Cadigan, University of Michigan), UAS-sSpi (gift of B.Z. Shilo, Israel) and $h s-N^{E C N}$ (gift of S. Artavanis-Tsakonas, Yale University). AyGal4 UAS-GFP.S65T (Actin5C > y + > Gal4, UAS-GFP), hs-Gal4, UAS-DNEgfr, P\{GawB\}LL54 (lattice cell Gal4), hs-FLP12] on X were all obtained from the Bloomington Stock Center.

Live visualization of pupal retinae. Pupae were picked as white prepupae and aged for $22-24 \mathrm{~h}$ at $25^{\circ} \mathrm{C}$. The pupal case covering the eye was removed and the pupa was laid on its side on a mounting pad of normal Drosophila media containing an extra $2 \%$ agar and covered with a coverslip. The mounted pupa was placed on the stage of a Zeiss Axiovert 200 and kept humidified and at $23-25^{\circ} \mathrm{C}$ during the experiment. Imaging was performed with an oil-immersion $\times 40$ objective using a Xenon bulb and a GFP filter. For each time point, a Z-series was imaged. The Z-series was required because of the dome shape of the eye and because the focal plane changes as the volume of the pupa increases developmentally. Images were captured with a Quantix CCD camera (Photometrics Ltd) and ImagePro Plus Software (ImagePro) and were processed through one round of sharpening and one or two rounds of low pass filter prior to analysis using Metamorph Software (Universal Imaging). In most cases, a region from each focal plane in which the apical membranes were best viewed was cut out. The cut regions were flattened into one image. All cell counting was performed by hand. Live imaging of the pupal eye had no effect on the viability of the pupa nor did it lead to any visible change in the adult eye.
Immunohistochemistry of pupal retinae. Pupae were aged at $25^{\circ} \mathrm{C}$. Retinae were dissected into PBS and fixed in $4 \%$ paraformaldehyde/PBS and permeabilized in PBS $/ 0.2 \%$ Triton X-100. Primary antibodies were: anti-armadillo N27A1 (1:10, Developmental Studies Hybridoma Bank, DSHB), antibetagalactosidase 40-1a (1:10, DSHB), anti-cut (1:10, DSHB), anti-E(spl) (mAB $3231: 2$, Gift from S. Bray), anti-dpERK (1:200, Sigma) and anti-CC3 (1:200, Cell Signaling Technologies). For anti-dpERK immunostaining, retinae were dissected into PBS containing $1 \mu \mathrm{M}$ okadaic acid, incubated for $5 \mathrm{~min}$ and fixed in PBS/4\% paraformaldehyde for 30-45 min. Secondary antibodies were Alexa-conjugated (Molecular Probes). TUNEL labeling was as described ${ }^{21}$ except that FITC-dUTP was incorporated (Molecular Probes).

\section{Acknowledgements}

We thank Lee Bardwell, Rainer Brachmann and especially Ross Cagan for helpful discussions and critical reading of the manuscript; Paul Garrity for the membraneGFP flies and S. Bray, B. Shilo and K. Cadigan for reagents. The monoclonal antibodies were obtained from the Developmental Studies Hybridoma Bank developed under the auspices of the NICHD and maintained by The University of lowa, Department of Biological Sciences. This research was partially supported by $\mathrm{NIH}$ training grant HD-07029 (JPM) and by a Career Award in the Biomedical Sciences from the Burroughs Wellcome Fund (CBB).

1. Jacobson MD, Weil M, Raff MC. Programmed cell death in animal development. Cell 1997; 88: $347-354$

2. Vaux DL. CED-4 - the third horseman of apoptosis. Cell 1997; 90: 389-390.

3. Abud HE. Shaping developing tissues by apoptosis. Cell Death Differ 2004; 11: 797-799.

4. Baehrecke EH. How death shapes life during development. Nat Rev Mol Cell Biol 2002; 3 779-787.

5. Hipfner DR, Cohen SM. Connecting proliferation and apoptosis in development and disease. Nat Rev Mol Cell Biol 2004; 5: 805-815.

6. Rusconi JC, Hays R, Cagan RL. Programmed cell death and patterning in Drosophila. Cell Death Differ 2000; 7: 1063-1070.

7. Brachmann CB, Cagan RL. Patterning the fly eye: the role of apoptosis. Trends Genet 2003; 19: 91-96.

8. Twomey C, McCarthy JV. Pathways of apoptosis and importance in development. J Cell Mol Med 2005; 9: 345-359.

9. Cagan RL, Ready DF. The emergence of order in the Drosophila pupal retina. Dev Biol 1989; 136: 346-362.

10. Wolff T, Ready DF. Cell death in normal and rough eye mutants of Drosophila. Development 1991; 113: 825-839.

11. Lawrence PA, Green SM. Cell lineage in the developing retina of Drosophila. Dev Biol 1979; 71: 142-152.

12. Wolff T, Ready DF. Pattern formation in the Drosophila retina In: Bate M, Martinez-Arias A (eds). The Development of Drosophila melanogaster. Cold Spring Harbor Laboratory Press: CSH, 1993

13. Miller DT, Cagan RL. Local induction of patterning and programmed cell death in the developing Drosophila retina. Development 1998; 125: 2327-2335

14. Sawamoto K, Okano H, Kobayakawa Y, Hayashi S, Mikoshiba K, Tanimura T. The function of argos in regulating cell fate decisions during Drosophila eye and wing vein development. Dev Biol 1994; 164 (1): 267-276.

15. Schweitzer R, Howes R, Smith R, Shilo BZ, Freeman M. Inhibition of Drosophila EGF receptor activation by the secreted protein Argos. Nature 1995; 376 (6542): 699-702.

16. Freeman M. Reiterative use of the EGF receptor triggers differentiation of all cell types in the Drosophila eye. Cell 1996; 87: 651-660.

17. Bergmann A, Agapite J, McCall K, Steller H. The Drosophila gene hid is a direct molecular target of Ras-dependent survival signaling. Cell 1998; 95: 331-341.

18. Kurada $\mathrm{P}$, White K. Ras promotes cell survival in Drosophila by downregulating hid expression. Cell 1998; 95: 319-329.

19. Cagan RL, Ready DF. Notch is required for successive cell decisions in the developing Drosophila retina. Genes Dev 1989; 3: 1099-1112.

20. Yu SY, Yoo SJ, Yang L, Zapata C, Srinivasan A, Hay BA et al. A pathway of signals regulating effector and initiator caspases in the developing Drosophila eye. Development 2002; 129: 3269-3278.

21. Cordero J, Jassim O, Bao S, Cagan R. A role for wingless in an early pupal cell death event that contributes to patterning the Drosophila eye. Mech Dev 2004; 121: 1523-1530.

22. Baker NE, Yu SY. The EGF receptor defines domains of cell cycle progression and survival to regulate cell number in the developing Drosophila eye. Cell 2001; 104: 699-708

23. Cadigan KM, Nusse R. Wingless signaling in the Drosophila eye and embryonic epidermis. Development 1996; 122: 2801-2812.

24. Jennings $B$, Preiss A, Delidakis $C$, Bray $S$. The Notch signalling pathway is required for enhancer of split bHLH protein expression during neurogenesis in the Drosophila embryo. Development 1994; 120: 3537-3548. 
25. Struhl G, Basler K. Organizing activity of wingless protein in Drosophila. Cell 1993; 72 $527-540$.

26. McGuire SE, Roman G, Davis RL. Gene expression systems in Drosophila: a synthesis of time and space. Trends Genet 2004; 20: 384-391.

27. Hsiung F, Griffis ER, Pickup A, Powers MA, Moses K. Function of the Drosophila TGFalpha homolog Spitz is controlled by Star and interacts directly with Star. Mech Dev 2001; 107: 13-23.

28. Kumar JP, Hsiung F, Powers MA, Moses K. Nuclear translocation of activated MAP kinase is developmentally regulated in the developing Drosophila eye. Development 2003; 130 3703-3714.
29. Kumar JP, Tio M, Hsiung F, Akopyan S, Gabay L, Seger R et al. Dissecting the roles of the Drosophila EGF receptor in eye development and MAP kinase activation. Development 1998; 125: 3875-3885.

30. Major RJ, Irvine KD. Influence of Notch on dorsoventral compartmentalization and actin organization in the Drosophila wing. Development 2005; 132: 3823-3833.

31. Crowner D, Le Gall M, Gates MA, Giniger E. Notch steers Drosophila ISNb motor axons by regulating the Abl signaling pathway. Curr Biol 2003; 13: 967-972.

32. Lowell S, Watt FM. Delta regulates keratinocyte spreading and motility independently of differentiation. Mech Dev 2001; 107: 133-140.

33. Giniger E. A role for Abl in Notch signaling. Neuron 1998; 20: 667-681.

Supplementary Information accompanies the paper on Cell Death and Differentiation website (http://www.nature.com/cdd) 